\title{
Focusing on materials
}

ULTRA FIELD-EMISSION SEM

EO

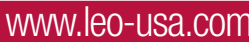

Ultra-high-resolution imaging

LEO Electron Microscopy Group introduce a family of ULTRA field-emission scanning electron microscopes that provide ultra-high-resolution topographic and compositional imaging, with an analytical specimen chamber. A fully integrated backscattered electron detector, EsB (energy and angleselected backscattered), is positioned in the GEMINI electron optical column directly above the secondary electron (SE) detector. Real-time simultaneous imaging is offered with both secondary and backscattered electrons without compromising the versatility of the equipment. Backscattered electrons are less sensitive

AXIOSKOP 2 MAT UPRIGHT MICROSCOPES

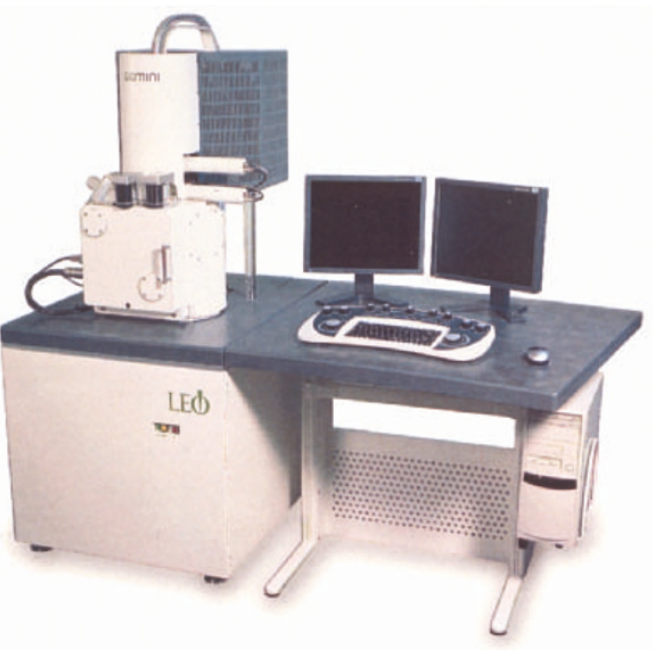

to charging and edge contrast, allowing clear imaging of boundaries and features in semiconductor and nanoscale technology. The EsB detector has a filtering grid that prevents secondary electrons from entering the EsB detector, and enables the production of highresolution images of charging specimens. The ULTRA offers new imaging capabilities for such as ceramics, catalysts, plastics, glass and nanoparticle manufacturing. Carl Zeiss

\section{www.zeiss.com/micro}

High-performance optics

The new Axiosxkop 2 MAT microscope from Carl Zeiss MicroImaging aims to meet the demands of materials microscopy research, quality assurance and failure analysis. The improved

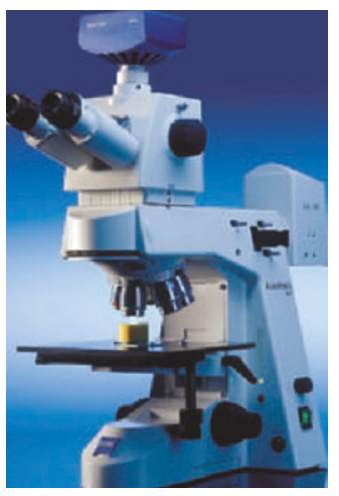

reflected-light beam path and high-performance optics offer better micrograph images. The motorized version has an automated reflector turret, z-drive, brightness control and switching mirror. The viewing angle of $20^{\circ}$ allows the viewing height to be varied by $50 \mathrm{~mm}$. New, high-performance materials objectives allow use of the new circular differential interference contrast (C-DIC) and total interference contrast techniques from Carl Zeiss, giving the possibility to recognize more details and to measure structures more precisely.
These notes are compiled in the Nature Materials office from information provided by the manufacturers.

C-DIC

Carl Zeiss www.zeiss.com/materials

Orientation-independent imaging

The new C-DIC technique from Zeiss uses circularly polarized light, unlike conventional DIC, to solve problems of insufficient contrast observed in classical brightfield and darkfield microscopy. This means that fine surface structures can be observed with high contrast and resolution simultaneously, independent of orientation. The C-DIC prism is inserted into the compensator mount of

\section{EVO SERIES SEMS} LEO wuw.leo-usa.com

Wide pressure-range capability

LEO announce a new EVO series of SEMs that have improved capabilities of imaging with extended variable pressure (XVP) within a range of 1 to $750 \mathrm{~Pa}$ using water vapour or air. X-ray analysis uses a combination of $8.5 \mathrm{~mm}$ working distance and a take-off angle of $35^{\circ}$. the microscope, and no stage or object rotation is required. The circularly polarized light ensures homogeneous illumination for improved image contrast with fully opened illumination aperture. Fixed mechanical and scanning stages can be used for higher throughput in defect analysis. Different system configurations are available by combining upright and inverted Zeiss microscopes with their AxioCam digital camera and Axio Vision software.

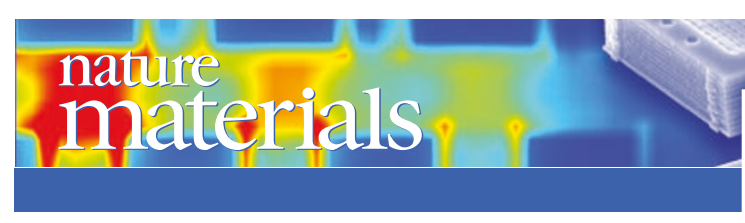

New on the Market

The 'New on the Market' section of Nature Materials is designed to give our readers access to the latest technologies, products and senvices available in the highly competitive materials science and engineering fields. We would like to invite you to send your Press Release Information about your new technologies, products and services for FREE inclusion in the features section 'New on the Market'.

For further information please contact Gerard Preston, Senior European Advertising Manager, by email at g.preston@nature.com 